\title{
Energy and Centrality Dependence of Chemical Freeze-out Parameters from Model Calculations
}

\author{
Lokesh Kumar* \\ Kent State University, \\ Kent, $\mathrm{OH} 44242$ \\ E-mail: lokeshercf.rhic.bnl.gov
}

\begin{abstract}
One of the main goals of heavy-ion collision experiments is to study the structure of the QCD phase diagram. The QCD phase diagram is typically plotted as temperature $(T)$ vs. baryon chemical potential $\left(\mu_{B}\right)$. The statistical thermal model THERMUS compared to experimental data provides chemical freeze-out parameters such as temperature, baryon chemical potential and strangeness saturation factor $\left(\gamma_{s}\right)$. However, the values of these parameters depend on models and their underlying assumptions, such as the nature of the ensemble used, particle ratios vs. particle yields, and the treatment of feed-down contributions to particle yields. In these proceedings, we report on a systematic study of chemical freeze-out parameters using THERMUS, as a function of collision centrality and collision energies $\left(\sqrt{s_{N N}}=7.7-200 \mathrm{GeV}\right)$. These studies are performed with the string melting version of A Multi-Phase Transport (AMPT) model. A comparison is presented of freeze-out parameters between grand-canonical vs. strangeness canonical ensembles, particle yields vs. ratios, with and without feed-down contributions to the particle yields. The main aim is to evaluate the sensitivity of the thermal model fits to various model assumptions. This is an important study for understanding corresponding experimental results from the beam energy scan program at RHIC.
\end{abstract}

8th International Workshop on Critical Point and Onset of Deconfinement,

March 11 to 15, 2013

Napa, California, USA

\footnotetext{
${ }^{*}$ Speaker.
} 


\section{Introduction}

One of the goals of heavy-ion collision experiments is to study the QCD phase diagram. The QCD phase diagram is generally represented by temperature $(T)$ versus baryon chemical potential $\left(\mu_{B}\right)$. Study of hadron production plays an important role in understanding the dynamics of relativistic collisions. The statistical thermal model has been extremely successful in describing the hadron multiplicities observed in these relativistic collisions. The statistical model or THERMUS uses experimental yields (or ratios) as input and provides the corresponding chemical freeze-out temperature and baryon chemical potential [1].

Recently, the STAR experiment has presented the centrality dependence of the freeze-out parameters using the Beam Energy Scan (BES) data. It is observed that at lower energies $\left(\sqrt{s_{N N}} \sim 7.7\right.$ and $11.5 \mathrm{GeV}$ ), the $T$ and $\mu_{B}$ vary with centrality [2]. Interestingly, for Grand-Canonical Ensemble (GCE), the temperature seems to decrease while going from central to peripheral collisions. On the other hand, the temperature increases while going from central to peripheral collisions when the Strangeness-Canonical Ensemble (SCE) is used. The baryon chemical potential decreases towards peripheral for both GCE and SCE.

In view of these observations, it is of interest to study the centrality and energy dependence of freeze-out parameters using transport models such as A Multi-Phase Transport (AMPT) model [3]. For this study, the string-melting version of AMPT is used. The particle yields $(d N / d y)$ at midrapidity $(|y|<0.5)$ are obtained for $\pi^{ \pm}, K^{ \pm}, p(\bar{p}), \Lambda(\bar{\Lambda}), K_{S}^{0}$, and $\Xi(\bar{\Xi})$ particles at $\sqrt{s_{N N}}=7.7$, $11.5,39$, and $200 \mathrm{GeV}$. The errors on yields are assumed to be of the order of $10 \%$ for realistic comparison with experimental results. The centrality classes used for this study are $0-5 \%, 5-10 \%$, $10-20 \%, 20-30 \%, 30-40 \%, 40-50 \%, 50-60 \%, 60-70 \%$, and 70-80\%. The THERMUS model is used for extracting chemical freeze-out parameters using both Grand-Canonical and StrangenessCanonical ensembles. The freeze-out parameters are extracted by using particle ratios as well as yields as input to the THERMUS model.

\section{Results and Discussions}

Figure 1 shows the variation of chemical freeze-out temperature and baryon chemical potential for different energies from 7.7 to $200 \mathrm{GeV}$ and different centralities. Top panels represent results obtained by using particle ratios from AMPT as inputs to the THERMUS model for GCE (left) and SCE (right) while bottom panels represent results obtained by using particle yields from AMPT as input to THERMUS. Both $T$ and $\mu_{B}$ show similar behavior as a function of centralities at all energies. Both decrease from central to peripheral collisions for all the cases studied. This is opposite to what was observed in the RHIC BES data, where for Strangeness-Canonical Ensemble, the temperature increases from central to peripheral collisions.

The comparison of freeze-out parameters obtained by using different inputs such as particle ratios and particle yield to THERMUS suggests that the temperature values are similar and lie within $5 \%$ for these cases. However, $\mu_{B}$ values could differ by a maximum of $20 \%$ between the two cases for SCE. When different ensembles (GCE vs. SCE) are used, the temperature values again lie within $5 \%$ whereas $\mu_{B}$ differ by $\sim 20 \%$ between GCE and SCE if yields are used as input to THERMUS. 

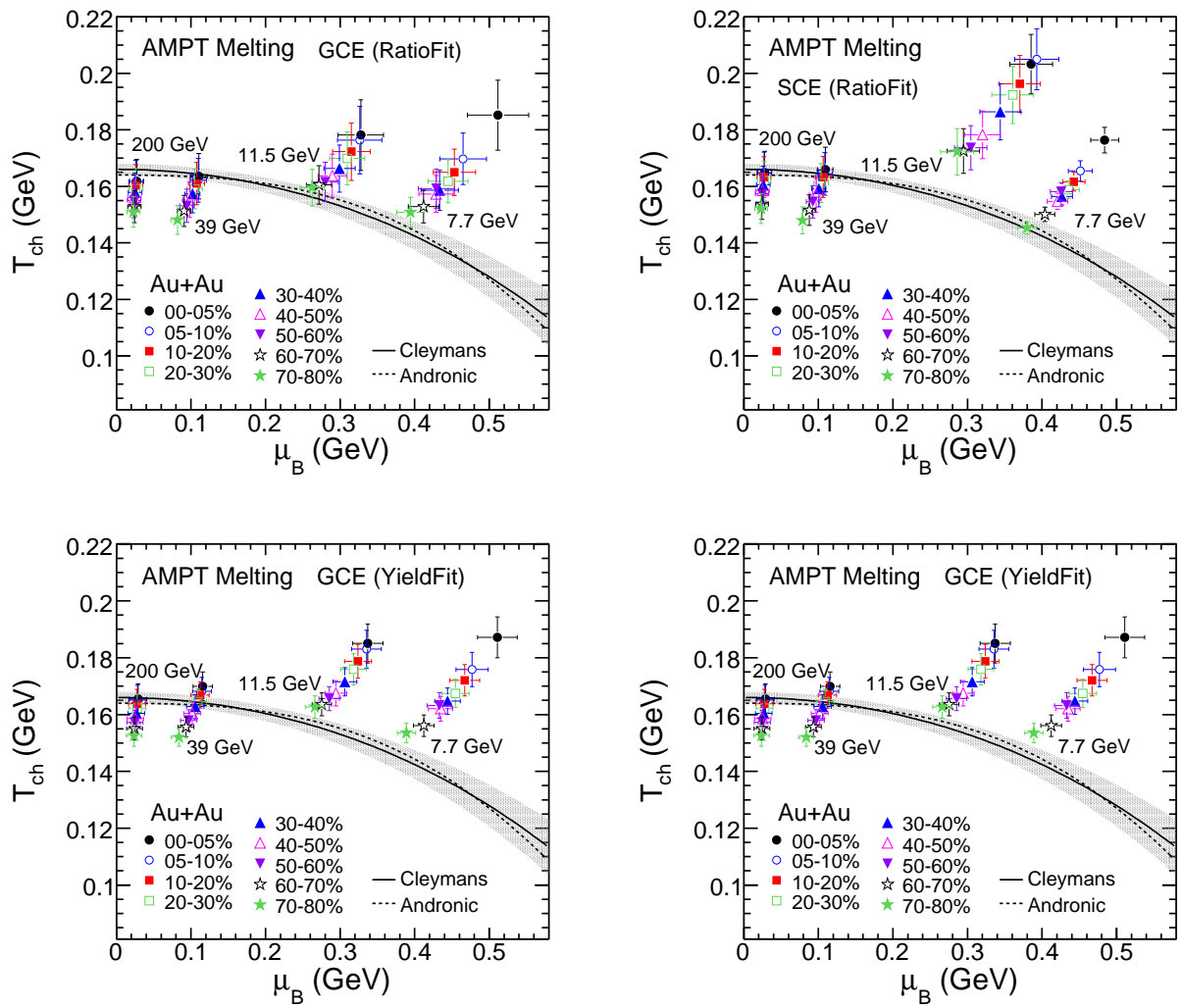

Figure 1: Top panels: Temperature vs. baryon chemical potential using particle ratios from AMPT as inputs to THERMUS model for GCE (left) and SCE (right). Bottom panels: Similar plots but obtained using particle yields from AMPT as inputs to THERMUS. Results are shown for beam energies from 7.7 to 200 $\mathrm{GeV}$ and for different centralities.

\section{Feed-down Effect}

In THERMUS, there is an option to switch OFF or ON the decay channels. This option can be used to test the effect of feed-down contribution on the extracted freeze-out parameters. We consider the weak-decay feed-down contribution from $\Lambda(\bar{\Lambda})$ to $p(\bar{p})$. For this purpose, Au+Au $200 \mathrm{GeV}$ from AMPT string melting is used. The $\Lambda$ and $\bar{\Lambda}$ are decayed respectively to $p$ and $\bar{p}$. The Grand-Canonical approach is used in THERMUS with input as the following particle ratios: $\pi^{-} / \pi^{+}, K^{-} / K^{+}, \bar{p} / p$, and $\bar{p} / \pi^{-}$. The feed-down contribution from $\Lambda$ to proton and $\bar{\Lambda}$ to $\bar{p}$ in THERMUS with default settings for $\mathrm{Au}+\mathrm{Au} 200 \mathrm{GeV}$ are of the order of $24 \%$. From AMPT, feeddown from $\Lambda$ to proton is $\sim 13 \%$ while that from $\bar{\Lambda}$ to $\bar{p}$ is $\sim 22 \%$. We study three cases: case 1 (No feed-down contribution from $\Lambda(\bar{\Lambda})$ to $p(\bar{p})$ in both AMPT and THERMUS), case 2 ( included feed-down contribution from $\Lambda(\bar{\Lambda})$ to $p(\bar{p})$ both in AMPT and THERMUS), and case 3 (feed-down contribution is included in both AMPT and THERMUS but the contributions in THERMUS are modified so that both AMPT and THERMUS have similar feed-down contributions from $\Lambda(\bar{\Lambda})$ to $p(\bar{p})$ ). Figure 2 shows the results for the three cases for temperature and baryon chemical potential vs. $N_{\text {part }}$. Both $T$ and $\mu_{B}$ show closer agreement for case 1 and case 3 . The $\mu_{B}$ values show large 

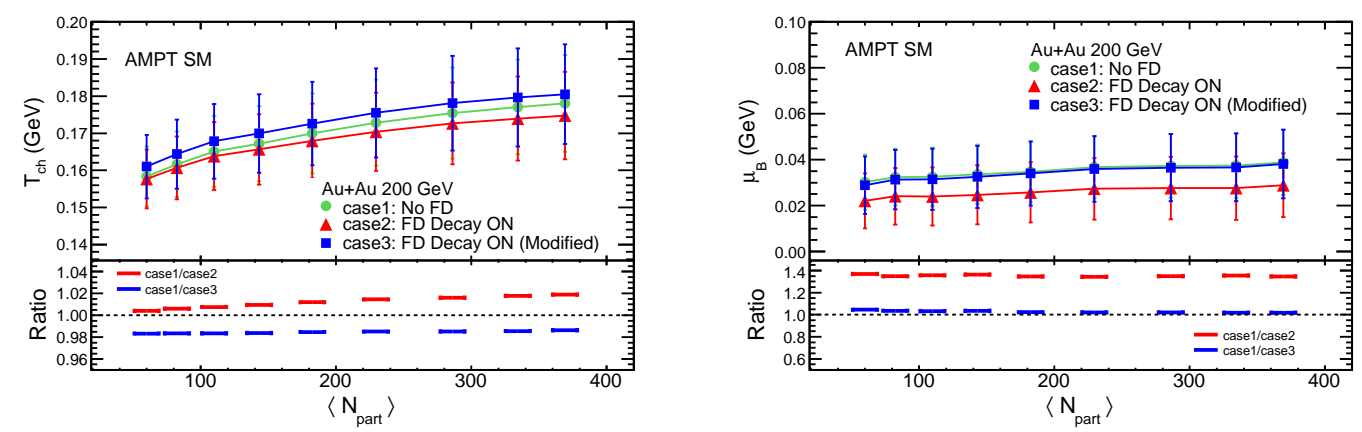

Figure 2: Top panels: Temperature (left) and baryon chemical potential (right) vs. $N_{\text {part }}$ for three different cases in $\mathrm{Au}+\mathrm{Au} 200 \mathrm{GeV}$ using AMPT string melting. Bottom panels: Ratios of case $1 / \mathrm{case} 2$ and case $1 /$ case 3 . See text for details.

difference if the feed-down is not taken care of properly in THERMUS (such as in case 2).

\section{Summary}

We have presented the energy and centrality dependence of freeze-out parameters from the AMPT model with a string melting scenario. Freeze-out parameters are extracted using THERMUS and compared for different ensembles (GCE vs. SCE) as well as for different inputs to THERMUS (particle yields vs. particle ratios). The $T$ and $\mu_{B}$ decrease from central to peripheral collisions for all these cases in contrast to what has been observed in STAR data, where $T$ increases from central to peripheral collisions in SCE. For both GCE vs. SCE and particle yields vs. ratios inputs, $T$ values lie within $5 \%$ while $\mu_{B}$ may differ by a maximum of $20 \%$. The effect of weak decay feed-down (from $\Lambda(\bar{\Lambda})$ to $p(\bar{p})$ ) on the extracted freeze-out parameters is also presented. THERMUS can be tuned to adjust the feed-down contributions according to data (AMPT in this case). The difference in feed-down contribution between data (AMPT in this case) and THERMUS is reflected in the extracted $\mu_{B}$ values.

Acknowledgement: We would like to thank Prof. D. Keane, N. Ming, Prof. B. Mohanty, Prof. N. $\mathrm{Xu}$, and STAR Collaboration for the Physics discussions. This work is supported by DOE grant DE-FG02-89ER4053.

\section{References}

[1] A. Andronic et al., Nucl. Phys. A 772, 167 (2006); J. Cleymans et al., Phys. Rev. C 73, 034905 (2006); F. Becattini et al., Phys. Rev. C 73, 044905 (2006); J. Cleymans et al., Comp. Phys. Comm. 180, 84 (2009).

[2] L. Kumar (for the STAR Collaboration) Nucl. Phys. A 904, 256c (2013); S. Das (for the STAR Collaboration) Nucl. Phys. A 904, 891c (2013).

[3] Z. W. Lin et al., Phys. Rev. C 72, 064901 (2005); Z. W. Lin et al., Phys. Rev. C 64, 011902 (2001); B. Zhang et al., Phys. Rev.C 61, 067901 (2000). 